\title{
BMJ Open Cohort protocol: Guangzhou High-Risk Infant Cohort study
}

\author{
Pian $\mathrm{Hu}$ (D) , ${ }^{1}$ Azhu Han, ${ }^{1}$ Yan Hu, ${ }^{1}$ Yuqi Wen, ${ }^{1}$ Jingjing Liang, ${ }^{1}$ Wanqi Xiao, ${ }^{1}$ \\ Suifang Lin, ${ }^{1}$ Yanyan Song, ${ }^{1}$ Xuying Tan, ${ }^{1}$ Xiaopeng Zhao, ${ }^{2}$ Haipeng Dong, ${ }^{1}$ \\ Qianyun Liu, ${ }^{1}$ Huayan Zhang, ${ }^{2,3}$ Li Tao, ${ }^{2}$ Yuan Yuan ${ }^{2}$
}

To cite: Hu P, Han A, Hu Y, et al. Cohort protocol: Guangzhou High-Risk Infant Cohort study. BMJ Open 2020;10:e037829. doi:10.1136/ bmjopen-2020-037829

- Prepublication history for this paper is available online. To view these files, please visit the journal online (http://dx.doi. org/10.1136/bmjopen-2020037829).

$\mathrm{PH}$ and $\mathrm{AH}$ contributed equally.

$\mathrm{PH}$ and $\mathrm{AH}$ are joint first authors.

Received 19 February 2020

Revised 26 June 2020

Accepted 06 July 2020

Check for updates

(C) Author(s) (or their employer(s)) 2020. Re-use permitted under CC BY-NC. No commercial re-use. See rights and permissions. Published by BMJ.

${ }^{1}$ Department of Child Health Care, Guangzhou Women and Children's Medical Center, Guangzhou Medical University, Guangzhou, China ${ }^{2}$ Neonatal Unit, The Neonatal Medical Center, Guangzhou Women and Children's Medical Center, Guangzhou Medical University, Guangzhou, China ${ }^{3}$ Division of Neonatology, Children's Hospital of Philadelphia, Philadelphia, Pennsylvania, USA

Correspondence to Professor Yanyan Song; yansong84@126.com

\section{ABSTRACT}

Introduction Despite the increase in the survival rate of high-risk infants (HRls) worldwide, the prevalence of motor and neurodevelopmental sequelae in such newborns has not shown concomitant improvement. Meanwhile, there are few cohorts that explore factors related to the development of HRIs in China. Therefore, the Guangzhou High-Risk Infant Cohort (GHRIC) has been designed to examine the complex relationships among a myriad of factors influencing growth and development in such children.

Methods and analysis The GHRIC study is a prospective cohort study that by the year 2023 will enrol an estimated total of 3000 HRIs from Guangzhou Women and Children's Medical Center (GWCMC) in Guangzhou, China. This study is designed to assess the growth and cognitive characteristics of HRls and the risk factors affecting their development and prognoses. Data on risk factors, neurodevelopmental and cognitive-function evaluations, laboratory results, and specimens will be collected and analysed. Information on perinatal and clinical interventions for these infants will also be recorded during regular follow-up visits until age 6 .

Ethics and dissemination The protocol for this study has been approved by the Research Ethics Committee of GWCMC, which accepted responsibility for supervising all of the aspects of the study (No. 2017102712). Study outcomes will be disseminated through conference presentations, peer-reviewed publications, the Internet and social media.

Trial registration number ChiCTR-EOC-17013236

\section{INTRODUCTION}

High-risk infants (HRIs) are commonly defined as (1) preterm infants; (2) infants with extremely low birth weight (ELBW) or who are small for gestational age (SGA); (3) development borderline children (DBC), defined as having central motor function that lags for more than 2 to 3 months, abnormal muscle tone and reflex, or abnormal posture; ${ }^{1}$ and (4) neonates with special healthcare needs or dependence on life support. ${ }^{23}$ Despite revolutionary changes in medical intervention that have ensured the survival of many HRIs worldwide, the prevalence of major and subtle sensory, cognitive,

\section{Strengths and limitations of this study}

In the context of high-risk infants (HRIs), the Guangzhou High-Risk Infant Cohort (GHRIC) study is particularly important, given the high prevalence of such infants and their possible long-term adverse health outcomes.

- The GHRIC study would enable us to understand the growth and developmental patterns of HRIs and provide suggestions for optimising clinical interventions.

- The GHRIC study could serve as a model to help facilitate research in this area by developing a management system to provide accessible, low-cost and feasible interventions for HRIs.

- High-frequency sampling will directly result in bias due to missing data and protocol deviations, as it is expected that some women will not be able to provide all per-protocol samples.

motor and neurodevelopmental sequelae, both short and long term, in HRIs has not shown concomitant improvement. ${ }^{4}$

Many investigators have identified a significant proportion of premature infants as having behaviours consistent with the symptoms of autism spectrum disorders (ASDs). In addition, recent findings have shown that infants with lower gestational ages presented crying, eating, and sleeping problems, indicating early regulatory disorders. In some cases, HRIs may have different degrees of disability (such as dyskinesia, developmental delay, language disorders, and behavioural abnormalities) in the future, depending on the severity of their conditions. ${ }^{5-8}$

Both maternal and infant risk factors could lead to the occurrence of HRIs. Risk factors for HRIs are commonly found in their mothers. Maternal age has been established as an important influencing factor for highrisk pregnancy, along with negative socioeconomic factors, low or high body mass index, history of diabetes, hypertension, smoking, alcohol abuse, exposure to air pollution and previous adverse obstetric complications. ${ }^{910}$ 
There are many cohorts for preterm infants overseas, such as the Extreme Premature Infant Cohort at the University College of London, UK (EPICure) $;^{11}$ the preterm-children cohort in Paris, France (L'Etude épidémiologique sur les petits âges gestationnels (Epipage)) $;^{12}$ the Project On Preterm and Small-for-gestational-age infants (POPS) in the Netherlands; ${ }^{13}$ and the preterm birth infant cohort in Nova Scotia, Canada. ${ }^{14}$ These cohorts have been followed up for many years, and many important conclusions have been drawn from the correlation between preterm birth and such factors as pulmonary function, neurodevelopment, learning ability, ASDs and the tendency toward hyperactivity.

At present, there are few cohorts for HRIs in China. One such cohort, which has a 12-month follow-up period, studies the effects of mobile healthcare on the health of HRIs (ChiCTR-IOC-17011521). Others include a multicentre cohort study on preterm infants $>32$ weeks in Shanghai (ChiCTR1900026853), a cohort of premature infants with very low birth weight (VLBW) and ELBW in Fujian Province (ChiCTR-INR-17010454), and a VLBW preterm child cohort in Taiwan. ${ }^{15}$ Each of these cohorts mainly focusses on a particular type of HRI; it is rare for a single cohort study to include several high-risk conditions, such as preterm birth, low birth weight (LBW), and multiple birth. The cohorts mentioned above can be validated at the Chinese Clinical Trial Register (http://www. chictr.org.cn/index.aspx).

The GHRIC study's protocol included the same management service process for premature infants as recommended in the healthcare guidelines for HRIs in China, including ultrasonography, blood test and growth monitoring at some fixed time (table 1). Due to the limited medical resources in China, not all the HRIs receive the immediate medical attention they need to ensure their health. We followed the guidelines to guarantee that the children in our cohort received the medical services they need, while adding an extra neuropsychologicaldevelopment examination to explore the special development patterns of HRIs. However, parental compliance during the regular management service process of HRIs was not very good, and the information collected beforehand was not complete or comprehensive. Therefore, a cohort following HRIs at a fixed time is necessary.

Guangzhou is one of the largest and most developed cities in China, with a large number of infants at risk of premature birth and LBW, a high prevalence of asthma in children $(6.9 \%)^{16}$ and an increasing incidence of congenital heart disease ${ }^{17}$ and birth defects. ${ }^{18}$ Therefore, Guangzhou is a suitable research site to explore possible factors affecting the prognoses, early feeding patterns and growth patterns of HRIs in China. ${ }^{19}$ Guangzhou Women and Children's Medical Center (GWCMC) is home to the Guangdong Early Childhood Development Center and Application Engineering Technology Research Center and is equipped with an international-standard, millionlevel biological-sample bank. The GWCMC assembled the Guangzhou High-Risk Infant Cohort (GHRIC) to examine factors that promote the healthy growth of HRIs. As the 'two-child policy' has been adopted in China, the number of advanced-age parturient women has increased year by year. The information we collect from this cohort will enable us to explore whether older maternal age and multiple birth are risk factors for HRIs. Overall, the HRI cohort will also provide a basis for optimisation of the high-risk-child management system and for future policies such as the 'three-child policy.'

\section{AIMS}

This project aims to improve the neurodevelopmental and cognitive outcomes of HRIs, including the following objectives:

1. Explore the disease spectrum of the main groups (preterm-birth and multiple-birth) of high-risk children.

2. Study the growth and development patterns of HRIs and the risk factors affecting their developmental process and prognoses.

3. Evaluate and examine current different interventions for HRIs.

4. Create a useful database of HRIs' growth, neurodevelopmental, cognitive and behavior-development outcomes that will facilitate future research activities.

\section{METHODS}

\section{Study design and setting}

The GHRIC study is a prospective cohort study on potential differences in growth, development, cognition and behaviour in HRIs before the age of 6 years, aiming to explore the disease spectrum of these children. It was designed and will be conducted by the Early Childhood Development Center and Application Engineering Technology Research Center of GWCMC, China.

\section{Study sample and enrolment}

\section{Study participants}

Previous studies and prior knowledge suggest that the main causes of HRIs include preterm birth, multiple birth, the infant being SGA or large for gestational age, hypoxic ischaemic encephalopathy, enrolment haemorrhage, hyperbilirubinaemia and maternal high-risk factors during pregnancy and delivery. In general, infants less than 3 months old with one or more high-risk factors will be enrolled at GWCMC, from the Early Childhood Development Center/Child Healthcare Clinic in GWCMC, China. The research coordinator will contact the parents or guardians when they bring their child to GWCMC for their regular healthcare visit. The routine healthcare plan for HRIs is to monitor their physical, psychological, behavioural, neurodevelopmental and cognitive health status. During the healthcare visits, infants will be examined by a doctor or physician for possible health problems to ensure early detection and intervention. The research coordinator will introduce basic information about the 


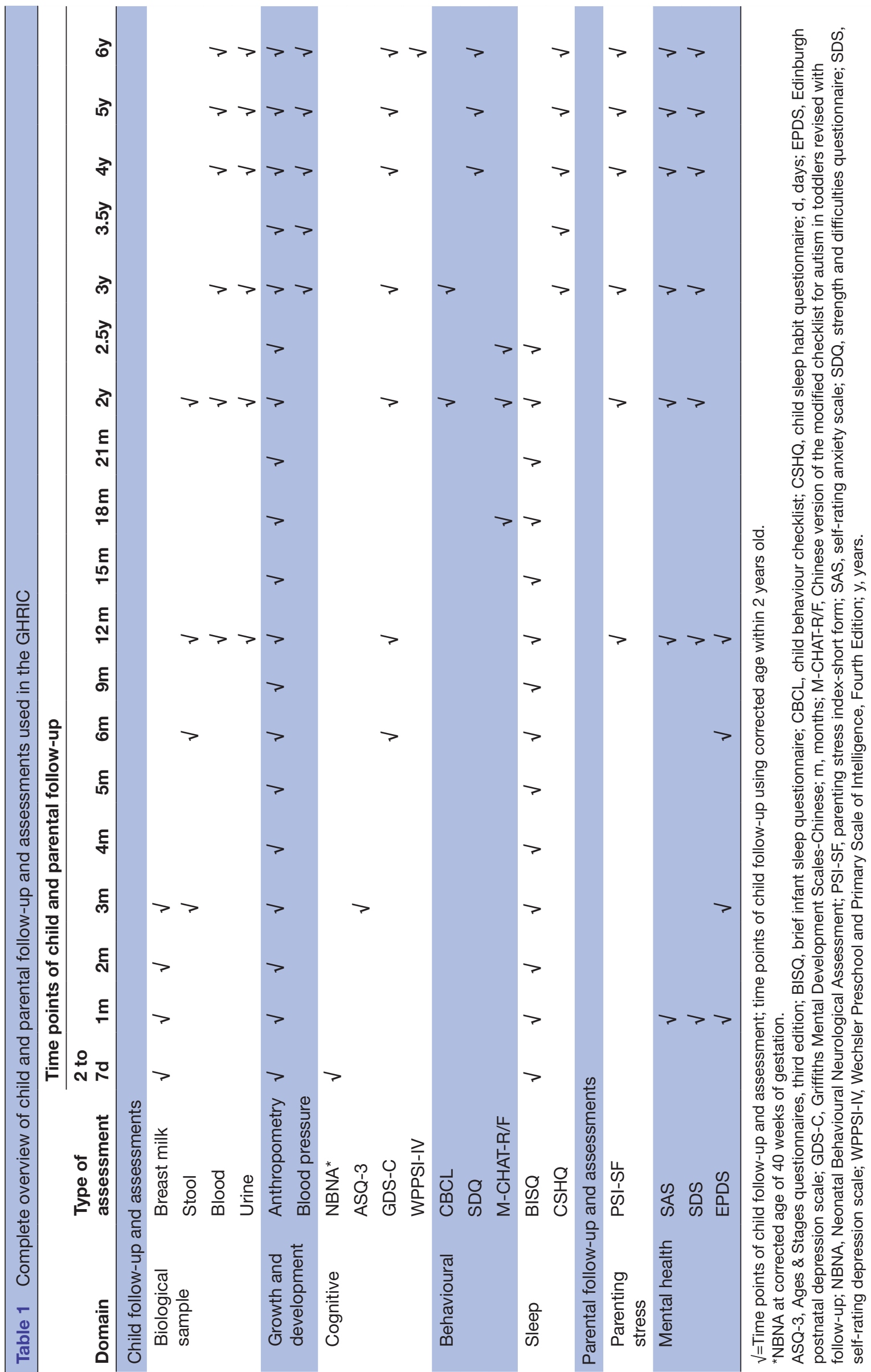

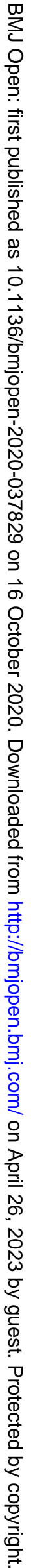


cohort to the parents or guardians, including its merits and inconveniences, while handing out a pamphlet with written information about the study. After being fully informed about the programme, families will be given a choice of whether to sign up for this study. Those who decide to enrol will be asked to sign a consent form; only then will the child be enrolled. Recruitment began in November 2019 and is expected to be completed in December 2023.

\section{Study procedure}

Figure 1 and table 1 show the details of the GHRIC study's follow-up procedure. After the parents or guardians sign the informed-consent form, each participant will be given a personal profile and assigned a seven-digit code for GHRICidentification. During the initial follow-up, parents or guardians will need to complete a baseline questionnaire that includes information on family, pregnancy and delivery. Thereafter, this study's follow-up schedule and procedure will be consistent with the Chinese follow-up guidelines for HRIs issued by the National Health Commission of the People's Republic of China http:// www.nhc.gov.cn/fys/mrgzdt/201703/d5656db4e43a49ff 9d14470864b0fb16.shtml. At each follow-up visit, parents or guardians will bring their child to the Early Childhood Development Center/Child Healthcare Clinic in the GWCMC for healthcare; during the visit, they will be directed to the GHRIC office for follow-up. The research coordinator will help them complete a follow-up checklist/questionnaire about the child's overall health status since the last follow-up and collect any necessary samples from the mother or child. Appointments for neurodevelopmental and cognitive assessments will be made for the child if necessary. In addition, the results of evaluation reports such as the Neonatal Behavioural Neurological Assessment (NBNA); Ages \& Stages Questionnaires, Third Edition (ASQ-3); Griffiths Mental Development Scales-Chinese (GDS-C); and Wechsler Preschool and Primary Scale of Intelligence, Fourth Edition (WPPSIIV) will be saved in the child's personal file. The current main method of completing the follow-up questionnaire is filling out the paper version.

\section{Inclusion criteria}

Inclusion criteria for the study are as follows:

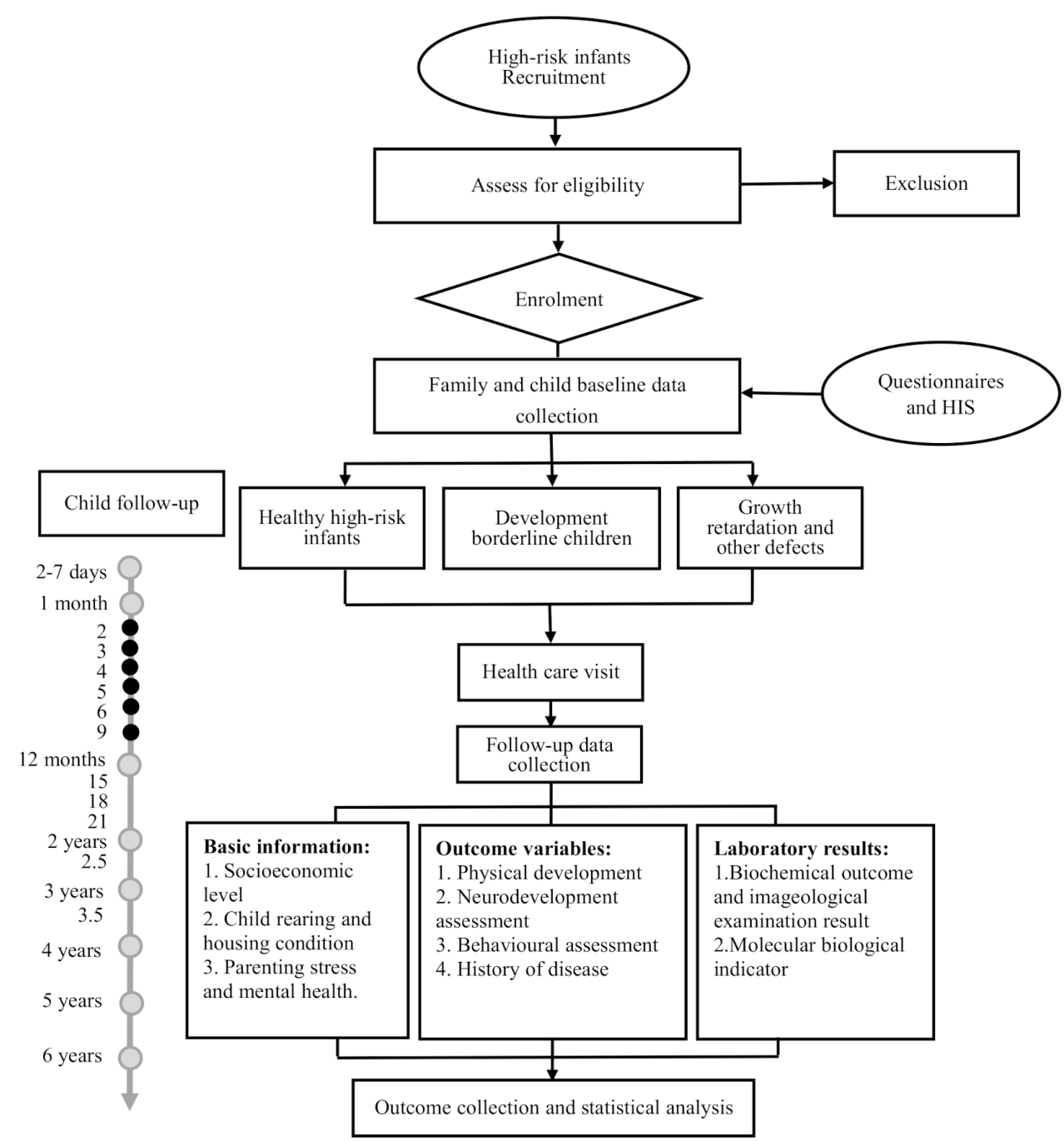

Figure 1 Recruitment process for the GHRIC. GHRIC, GuangzhouHigh-Risk Infant Cohort; HIS, hospital information system. 
1. HRIs, commonly defined as neonates with high-risk factors, including preterm infants $(<37$ weeks of gestational age), ELBW infants $(<1000 \mathrm{~g})$, SGA infants, DBC or infants dependent on life support.

2. Infants $<3$ months of age.

\section{Exclusion criteria}

Exclusion criteria for the study are as follows:

1. One or both parents or guardians are not registered as residents of Guangzhou.

2. Parents or guardians do not plan for their infant to receive long-term healthcare at GWCMC.

\section{Sample size}

GWCMC is one of the largest tertiary hospitals in China, with 18000 to 20000 annual deliveries of the 220000 total births in the Guangzhou municipality. ${ }^{20}$ Therefore, we expect to initially enrol 3000 GHRIC participants from GWCMC over a period of 4 years (2019 to 2023). This number will be sufficient to conduct longitudinal research on developmental disabilities and behavioural disorders of HRIs. The GHRIC study is primarily an epidemiological investigation without any predefined statistical hypothesis to test, making a priori sample size calculation unnecessary. ${ }^{21}$ In designing this study, we wished to ensure that we obtained the correct answers in the most efficient way, so that the work that we do will be worthwhile. Therefore, we will enrol enough HRIs to give our results adequate power, but not too many that we waste time gathering more data than we need. ${ }^{22}$

\section{Data collection}

In the GHRIC study, data collection will begin following consent and enrolment (figure 1 and table 1). During the first visit, the research coordinator will inform parents or guardians about the study before soliciting a signed informed consent and conducting the baseline assessment of the infant. Perinatal data will be collected by a member of the research team from the Clinical Data Center of GWCMC. The baseline information of the HRIs delivered at GWCMC is accessible from the hospital's information system. For infants born at other hospitals or institutions, baseline information will be extracted from their birth records and recorded in the HRIs management system for storage. Any medical records obtained from the hospital information system (HIS) will not be released without the permission of the principal investigator.

For this study, we will collect $5 \mathrm{~mL}$ blood samples, 3 to $4.5 \mathrm{~mL}$ stool samples and 100 to $200 \mathrm{mg}$ urine samples from the child, as well as $5 \mathrm{~mL}$ breast milk sample from the mother, at a specific time (table 1). The specimens will be stored at $-80^{\circ} \mathrm{C}$ until processing at the Clinical Biological Resource Bank of GWCMC. In this study, we will use blood samples to analyse gene methylation, glucolipid metabolism, hormones and micronutrients. Breast milk samples will be used for nutritional analysis; stool samples will be collected to analyse gut microbiota in high-risk children, such as the $16 \mathrm{~S}$ ribosomal RNA (rRNA) gene; urine samples will be used to analyse endocrine disruptors, including bisphenols $\mathrm{A}, \mathrm{F}$ and $\mathrm{S}$ (BPA, BPF, BPS); organophosphate esters (OPEs); and phthalate esters (PAEs). Additionally, all results of these tests will be recorded and made available to the parents or guardians.

Anthropometry of the enrolled children will be measured at each visit (table 1), including length/height, weight, head circumference, chest circumference, abdominal circumference, upper-arm circumference, skin fold thickness and other indicators. In addition, we will assess the child's neurodevelopmental and behavioural status during follow-up visits. Special cases will require MRI of the brain, which will also be recorded in our management system. Meanwhile, self-administered questionnaires will be used to collect information on socioeconomic status (SES) and other demographics, vitamin and mineral supplementation, and feeding strategies.

Maternal-baseline questionnaires, clinical assessments and anthropometry of the infants will be obtained at enrolment. Maternal information will include age, comorbidities/high-risk factors (preeclampsia, gestational diabetes, chorioamnionitis and in vitro fertilisation), country of birth, dietary preferences, smoking status, parity, gravidity, mode of delivery, antenatal-screening test results (eg, oral glucose tolerance test, coagulation test and maternal biochemistry), ultrasound findings, obstetric complications and medication prescriptions. In addition, paternal characteristics including social, demographic and lifestyle information will be collected.

\section{Interventional measurements}

During follow-up for the GHRIC study, high-risk children will receive timely and effective interventions and treatments depending on the doctor's orders, particularly those of GWCMC's neurology and rehabilitation departments. Interventional measures will generally include motor rehabilitation, language training, catch-up growth exercises, hand function promotion and oral muscle training. We will record intervention sessions in detail, including duration, frequency, adherence and any assessments done during the sessions, with the aim of systematically managing and evaluating both the short-term and long-term effects of these interventions in the future.

\section{Outcomes}

\section{Primary outcome}

The long-term perspective of this project is to find possible evidence and correct the delays in HRIs' physical, psychological, behavioural, neurodevelopmental and cognitive growth. The primary outcome is measured by (more details in table 1 ):

- Anthropometric measurement.

- Neurodevelopmental and cognitive assessments using the NBNA, ASQ-3, GDS-C and WPPSI-IV.

- Behavioural problems as measured by the Child Behaviour Checklist (CBCL); Strength and Difficulties Questionnaire (SDQ); Chinese version of the Modified Checklist for Autism in Toddlers, Revised 
with Follow-up (M-CHAT-R/F); Brief Infant Sleep Questionnaire (BISQ); and Childhood Sleep Habits Questionnaire (CSHQ).

\section{Secondary outcome}

Secondary outcome measures will encompass family parenting, environmental exposures and the health status of the HRIs, all of which are potentially related to the physical, psychological, behavioural, neurodevelopmental and cognitive health of these children. Secondary outcomes will be measured as follows:

- The family parenting environment, including the mental health of parents and parenting stress. We will measure these factors using the Self-rating Anxiety Scale (SAS), Self-rating Depression Scale (SDS), Edinburgh Postnatal Depression Scale (EPDS) and Parenting Stress Index-short form (PSI-SF).

- We will test for environmental exposures by analysing infants' stool, blood, and urine specimens and mothers' breast milk samples.

- Clinical notes from records will be extracted from the HIS and high-risk management system.

\section{Child follow-ups and assessments}

Neurodevelopmental and cognitive assessments

Infants' neurodevelopmental outcomes will be evaluated using the NBNA, which was formulated by Bao et $a l^{23}$ based on the Neonatal Behavioural Assessment Scale (NBAS) of Brazelton and Amiel-Tison, and is used to assess behavioural neurology in 3-day-old Chinese newborns (48 to 72 hours after birth). In this study, we will use the NBNA to evaluate neonatal behaviour at the corrected age of 40 weeks. The Chinese version of the ASQ- $3^{24} 25$ will be used to evaluate infants' neurodevelopment at the corrected age of 3 months.

We will assess the children's developmental states using the GDS-C ${ }^{26}$, which consists of six subscales: locomotor, personal-social, language, eye-hand coordination, performance and practical reasoning. The mean value of the six subscale scores is defined as the general quotient. At the corrected ages of 6,12 and 24 months, and the physical ages of $3,4,5$ and 6 years, the intelligence profile of each participant will be assessed using the GDS-C, which will be administered by a certified developmental paediatrician. Moreover, we will use the WPPSI-IV to test the cognitive development of children at age 6 years. The WPPSI mainly consists of the Verbal Comprehension Index, Visual-Spatial Index, and Full-Scale Intelligence Quotient scores. All reported scores will be standardised according to the WPPSI-IV user's manual. ${ }^{27}$

\section{Behavioural assessment}

Children's behavioural development will be assessed at 2 and 3 years of age using the $\mathrm{CBCL}^{28}$ and at 4 to 6 years of age using the Parent-report Strengths and Difficulties Questionnaire (SDQ-P). Parents will complete the CBCL/1.5 to 5, which is divided into two broad-band types of problems, including internalising (emotional reaction, anxiety/depression, somatic complaints and withdrawal) and externalising (aggressive behaviour and attention) problems. The $\mathrm{SDQ}^{29}$ was compiled by the American psychologist Robert Goodman in 1997 to assess the emotional and behavioural problems of children and adolescents. It has good reliability and validity, and is suitable for screening and evaluating emotional and behavioural problems in children and adolescents aged 3 to 16 years. ${ }^{30}$

Moreover, to facilitate early detection of and intervention for ASDs, we will administer the M-CHAT-R $/ \mathrm{F}^{31}$ to the children at ages 1.5, 2 and 2.5 years. M-CHAT-R/F is an effective tool for early detection of ASDs and is applicable for early screening in China, with adequate inter-rater reliability and test-retest reliability (intraclass correlation coefficients are 0.853 and 0.759 , respectively) ${ }^{31}$.

In this study, we will assess sleep duration and sleep disorders using the $\mathrm{BISQ}^{32}$ and the CSHQ ${ }^{33}{ }^{34}$ respectively. Parents or guardians will be instructed to recall the child's sleeping status during the past week. At the first stage (corrected age of $<2$ years), we will use the BISQ to characterise the infant's sleep quality. It has high sensitivity for evaluating sleep quality in young children. At the second stage (ages 2 to 6 years), the CSHQ will be used to assess general sleep quality. The CSHQ is a retrospective, 33-item, parent-reported questionnaire that evaluates sleep behaviours in school-aged children. The Chinese version of the CSHQ has been validated in a previous report ${ }^{34}$, which included eight domains: bedtime resistance, sleep onset delay, sleep duration, sleep anxiety, night waking, parasomnias, sleep dyspnoea and daytime sleepiness. In this cohort, parents or guardians will complete questionnaires covering their children's sleeping habits over the past week.

\section{Parental follow-up and assessments}

\section{Parenting stress}

The PSI-SF ${ }^{35}$ is a 36-item questionnaire designed to measure stress in the parent-child system. The PSI-SF consists of three subscales: parental distress, parent-child dysfunctional interaction and difficult child. Each item is rated on a 5-point Likert-type scale from 1 (strongly disagree) to 5 (strongly agree). The higher the subscale scores and PSI-SF total score, the greater the levels of stress. The Chinese version of the PSI-SF has demonstrated satisfactory reliability in clinical ${ }^{36}$ and nonclinical $^{37}$ settings. In the GHRIC study, parenting stress will be measured yearly.

\section{Mental health}

The Zung SAS $^{38}$ and SDS $^{39}$ will be used to assess anxiety and depression levels of children and caregivers, respectively. Each scale consists of 20 parameters, each of which is rated on a scale of 1 to 4 . The score for each parameter is then added and multiplied by 1.25 to obtain the final score. A high score $(\geq 50)$ on the SAS or SDS indicates a severe state of anxiety or depression, respectively. ${ }^{40}$ In the 
GHRIC study, anxiety and depression of parents or guardians will be assessed annually.

Additionally, we will use the $\operatorname{EPDS}^{41}$ to evaluate postpartum depression in mothers after childbirth. The EPDS contains 10 items, each scored on a 4-point scale (0 to 3) based on severity of symptoms. In the GHRIC study, we will use a validated Chinese version of the EPDS with appropriate linguistic and cultural contexts ${ }^{42}$ at $3,6,9$ and 12 months after delivery.

\section{Data management and monitoring}

High-risk infant management system

The HRI management system is used to manage patients at GWCMC, particularly those with risk factors during gestation and infancy. This system aims to monitor the growth, physical development, nutrition and neuropsychological development of infants with special needs and to promote regular monitoring of, detect underlying problems in, and provide timely check-ups for these children. It also acts as a systematic management platform for the implementation of GHRIC work.

\section{Quality control and supervision}

Each questionnaire will be evaluated by two auditors, who will check the completeness and reliability of each part of the questionnaire. For low-quality questionnaires, the reviewers will further contact the parents or guardians for a telephone re-interview within 1 week's time to complete and revise the questionnaire. For quality assurance, two individuals will separately perform data entry. Further, a logic check function was programmed into the data entry system beforehand to prevent entry errors. Data administrators will check the data regularly, and hospital investigators will be required to fill in omissions or correct possible errors. The quality control (QC) team, composed of members from the HRIs project workforce, will regularly conduct QC on outpatient follow-up electronic medical records and outpatient satisfaction surveys, and will conduct daily questionnaire registration, daily report verification and questionnaire reviews. Periodically, a random sample of the documented questionnaires selected from the archives will be re-entered and checked for quality.

\section{Queue maintenance and control of missed visits}

The enrolled patients will be assigned 20 follow-up visits from the corrected age of 3 months to 6 years; if a child misses a visit, the assessor will contact the parents or guardians and conduct the interview by phone. Additionally, our cohort has its own official WeChat account, which will be mainly used to post parenting tips, important notices or reminders for parents. We will also use this account to provide support and counsel for the parents or guardians of HRIs, which could increase the follow-up rate of the cohort.

\section{Statistical analysis}

For continuous variables, we will use means and SDs to present normally distributed data; for other data, we will use medians and IQRs. Numbers and percentages will be used for categorical variables. Continuous variables will be compared using the t-test or Mann-Whitney test, categorical variables using the $\chi^{2}$ test or Fisher's exact test. Linear/non-linear mixed-effects regression models and structural-equation models will be used to explore causal inference on the development of HRIs, including growth, development, cognition and behaviour. Additionally, we will perform longitudinal structural-equation modelling or growth curve analysis to evaluate the temporal effects of growth, neurodevelopment, behavioural development and even interventional measures for HRIs. All of the analyses performed in this study will be considered statistically significant at a level of 5\%, and $95 \%$ CIs will be calculated for all of the data.

\section{Ethics and dissemination}

The protocol for this study has been approved by the Research Ethics Committee of GWCMC, which has accepted responsibility for supervising all aspects of the study (No. 2017102712). All parents or guardians or caregivers of infants who agree to participate in the study will sign informed consent. The study protocol (2017-11-3) has been registered with the Chinese Clinical Trial Registry. Only the researchers associated with the study and the Ethics Committee would have access to the research data. The results of this study will be presented at both national and international conferences and be considered for publication in a peer-reviewed scientific journal. All the results presented in this study will be of group data; therefore, individual participants will not be identifiable.

\section{Patient and public involvement}

No parents or guardians has been or will be involved in the development of the plan for designing, conducting, reporting or implementing this study.

\section{DISCUSSION}

The GHRIC, a study specialising in HRIs, is also notable for being conducted in Guangzhou, as Guangzhou is one of the largest and most developed cities in China and has a large number of HRIs. Our aim is to study the overall development of HRIs as well as related factors such as nutrition and environmental exposures that might affect the growth and development of these children. The results of this study could provide guidance and researchbased support for formulating healthcare plans and policies for HRIs. With the adoption of the two-child policy in China, the prevalence of HRIs has been increasing in recent years. Although the technological advances that have improved perinatal/neonatal care and treatment and therefore increased the survival rate of HRIs exponentially, risks of short-term and long-term neurodevelopmental deficits still persist, particularly neurodevelopmental and behavioural problems. 
HRIs are more prone to develop complications such as neonatal necrotising enterocolitis, severe infections and neonatal respiratory distress syndrome, which increase the risk of extrauterine-growth restriction. Additionally, the growth and development of preterm infants before and after they reach full term further affects their short-term and long-term neurodevelopment. From full term to 4 months of age, greater weight gain (1.7 points) and linear growth (2.0 points) are associated with higher psychomotor development indices. ${ }^{43}$ According to the Taiwan Premature Infant Developmental Collaborative Study Group,${ }^{15}$ the prevalence of stunting is $15.8 \%, 16.9 \%$ and $12.0 \%$ at the corrected ages of 6,12 and 24 months, respectively. After potential confounding factors are controlled for, post-discharge stunted growth is significantly associated with poor neurodevelopmental outcomes. Neurodevelopmental alterations have been described in children born before 32 weeks of gestation in several cohorts worldwide, and these alterations concerned motor or sensory functions as well as emotional, behavioural and cognitive skills. ${ }^{4-48}$ Simoes $e t a l^{49}$ also demonstrated that compared with adequate-for-gestational-age full-term infants, both preterm infants with intrauterine-growth restriction and those with normal birth weights had altered neurodevelopmental trajectories. In Taiwan, a prospective, longitudinal, multicentre cohort study conducted from 2007 to 2011 indicated that most survivors $(87.6 \%)$ had major morbidities during hospitalisation and $79.4 \%$ of them received a follow-up evaluation after 2 years of age. Results showed that $39.7 \%$ of these infants had neurodevelopmental impairments, with incidence rates of $75 \%$, $65.2 \%, 49.5 \%, 39.5 \%$ and $32.8 \%$ for those born at 22, 23 , 24, 25 and 26 weeks, respectively. ${ }^{50}$ Therefore, systematic management of HRIs is necessary.

Moreover, HRIs face a broad range of neurodevelopmental sequelae, especially those affecting behaviour. For example, LBW and gestational age have been identified in several studies as important perinatal risk factors for disturbances in social interaction, communication and behaviour, as well as for psychoaffective disorders in adulthood. Recent studies suggest that many premature infants are at a higher risk of ASDs and attention deficit hyperactivity disorder (ADHD) ${ }^{51}$ Lower gestational age is associated with higher ADHD and ASD symptom rates. Males are identified as having higher rates of ADHD symptoms, and infants born into lower-SES families are associated with higher rates of ASD symptoms. Even school-aged very preterm children show higher degrees of ADHD and ASD symptoms, particularly difficulties in paying attention, socialising and communicating. ${ }^{52}$ Despite the progress made recently, our understanding of the behavioural and psychosocial health of HRIs remains limited.

Although the current understanding of risk factors of HRIs and how they interact is incomplete, perinatal factors are related to permanent neurosensory impairments such as cerebral palsy, blindness and deafness. Therefore, it is necessary to assemble an integrated, organised team to tackle HRI healthcare, schedule follow-ups, monitor growth and neurodevelopment, and provide detailed nutritional education and thorough inspection. This will improve development at this key early stage and reduce disability, thus improving the quality of life for HRIs. Hopefully, the GHRIC will contribute to better recognition of and prevention and intervention for HRIs, and achievement of optimal HRI healthcare.

Contributors $\mathrm{PH}$ and $\mathrm{AH}$ drafted the manuscript. YH, SL, XZ and YS designed and conceptualised the study. $\mathrm{PH}, \mathrm{AH}, \mathrm{YW}$ and $\mathrm{JL}$ carried out and revised the final version of the manuscript critically. XT, WX, HD, QL, HZ, LT and YY contributed to plan the study. All the authors contributed to revising and approved the final manuscript for submission.

Funding This work was supported by the Science and Technology Programme of Guangdong Province, China (No. 2017A020214002; funder approval no 2017A020214002). The anticipated completion date is 0ctober 2029.

\section{Competing interests None declared.}

Patient and public involvement Patients and/or the public were not involved in the design, or conduct, or reporting, or dissemination plans of this research.

Patient consent for publication Not required.

Provenance and peer review Not commissioned; externally peer-reviewed.

Open access This is an open access article distributed in accordance with the Creative Commons Attribution Non Commercial (CC BY-NC 4.0) license, which permits others to distribute, remix, adapt, build upon this work non-commercially, and license their derivative works on different terms, provided the original work is properly cited, appropriate credit is given, any changes made indicated, and the use is non-commercial. See: http://creativecommons.org/licenses/by-nc/4.0/.

ORCID iD

Pian Hu http://orcid.org/0000-0002-0525-8911

\section{REFERENCES}

1 Chen X, Fu G, Yang X, et al. Clinical study of early intervention in developmental borderline children. J Mod Clin Med 2008;34:264-5.

2 Cust AE, Darlow BA, Donoghue DA, et al. Outcomes for high risk New Zealand newborn infants in 1998-1999: a population based, national study. Arch Dis Child Fetal Neonatal Ed 2003;88:15F-22.

3 Song YY. A new systematic management model of perinatal high risk infants. JPM 2009;12.

4 Browne JV. Developmental care for high-risk newborns: emerging science, clinical application, and continuity from newborn intensive care unit to community. Clin Perinatol 2011;38:719-29.

5 Romeo DM, Leo G, Lapenta L, et al. Sleep disorders in low-risk preschool very preterm children. Sleep Med 2019;63:137-41.

6 Jones KM, Champion PR, Woodward LJ. Social competence of preschool children born very preterm. Early Hum Dev 2013;89:795-802.

7 Potijk MR, de Winter AF, Bos AF, et al. Higher rates of behavioural and emotional problems at preschool age in children born moderately preterm. Arch Dis Child 2012;97:112-7.

8 Wolke D, Schmid G, Schreier A, et al. Crying and feeding problems in infancy and cognitive outcome in preschool children born at risk: a prospective population study. J Dev Behav Pediatr 2009;30:226-38.

9 Waldenström U, Cnattingius S, Vixner L, et al. Advanced maternal age increases the risk of very preterm birth, irrespective of parity: a population-based register study. BJOG 2017;124:1235-44.

10 Australian Resuscitation Council, New Zealand Resuscitation Council. Planning for neonatal resuscitation and identification of the newborn infant at risk. Arc and NZRC guideline 2010. Emerg Med Australas 2011;23:424-5.

11 Balasubramanian S, Beckmann J, Mehta H, et al. Relationship between Retinal Thickness Profiles and Visual Outcomes in Young Adults Born Extremely Preterm: The EPICure@19 Study. Ophthalmology 2019;126:107-12.

12 Pinto Cardoso G, Houivet E, Marchand-Martin L, et al. Association of intraventricular hemorrhage and death with tocolytic exposure in preterm infants. JAMA Netw Open 2018;1:e182355.

13 Euser AM, Finken MJJ, Keijzer-Veen MG, et al. Associations between prenatal and infancy weight gain and BMI, fat mass, and fat 
distribution in young adulthood: a prospective cohort study in males and females born very preterm. Am J Clin Nutr 2005;81:480-7.

14 Vincer MJ, Allen AC, Joseph KS, et al. Increasing prevalence of cerebral palsy among very preterm infants: a population-based study. Pediatrics 2006;118:e1621-6.

15 Hsu C-T, Chen C-H, Lin M-C, et al. Post-Discharge body weight and neurodevelopmental outcomes among very low birth weight infants in Taiwan: a nationwide cohort study. PLoS One 2018;13:e0192574.

16 Chen Y, Wong GWK, Li J. Environmental exposure and genetic predisposition as risk factors for asthma in China. Allergy Asthma Immunol Res 2016;8:92-100.

17 Qu Y, Liu X, Zhuang J, et al. Incidence of congenital heart disease: the 9-year experience of the Guangdong registry of congenital heart disease, China. PLoS One 2016;11:e0159257.

$18 \mathrm{Yu}$ J, Zeng XQ, Zeng FL, et al. Analysis of 5597 cases of birth defects in Guangzhou. Chinese Journal of Woman and Child Health Research 2007;018:187-9.

19 Zhang J, Tian Y, Wang W, et al. Cohort profile: the Shanghai birth cohort. Int J Epidemiol 2019;48:21-21g.

20 Qiu X, Lu J-H, He J-R, et al. The born in Guangzhou cohort study (BIGCS). Eur J Epidemiol 2017;32:337-46.

21 Li L, Li K, An C, et al. Identification of risk factors affecting catch-up growth after infant congenital heart disease surgery: rationale and design of a multicentre prospective cohort study in China. BMJ Open 2019;9:e030084.

22 Jones SR, Carley S, Harrison M. An introduction to power and sample size estimation. Emerg Med J 2003;20:453-8.

23 Bao X, Yu R, Li Z, et al. Twenty-item behavioral neurological assessment for normal newborns in 12 cities of China 1991;104:742-6.

24 Wei M, Bian X, Squires J, et al. Studies of the norm and psychometrical properties of the ages and stages questionnaires, with a Chinese national sample 2015;53:913-8.

25 Bian X, Yao G, Squires J, et al. Translation and use of parentcompleted developmental screening test in Shanghai. J Early Childhood Res 2012;10:162-75.

26 Tso WWY, Wong VCN, Xia X, et al. The Griffiths development ScalesChinese (GDS-C): a cross-cultural comparison of developmental trajectories between Chinese and British children. Child Care Health Dev 2018;44:378-83.

27 WPPSI-IV. échelle d'intelligence de Wechsler pour enfants. ECPA, 2014.

28 Achenbach TM. Manual for the child behavior Checklist/2-3 and 1992 profile. Department of Psychiatry, University of Vermont, 1992.

29 Goodman R. The strengths and difficulties questionnaire: a research note. J Child Psychol

30 Vostanis P, PJCOiP V. Strengths and difficulties questionnaire: research and clinical applications. Curr Opin

31 Guo C, Luo M, Wang X, et al. Reliability and validity of the Chinese version of modified checklist for autism in toddlers, revised, with follow-up (M-CHAT-R/F). J Autism Dev Disord 2019;49:185-96.

32 Sadeh A. A brief screening questionnaire for infant sleep problems: validation and findings for an Internet sample. Pediatrics 2004;113:e570-7.

33 Owens JA, Spirito A, McGuinn M. The children's sleep habits questionnaire (CSHQ): psychometric properties of a survey instrument for school-aged children. Sleep 2000;23:1-9.
34 Li S-hui, Jin X-ming, Shen X-ming, et al. [Development and psychometric properties of the Chinese version of Children's Sleep Habits Questionnaire]. Zhonghua Er Ke Za Zhi.

35 Abidin R, Ona N. Parenting stress index. In: Professional manual. 3rd edn. Odessa, FL: Psychological Assessment Resources: Inc, 1995.

36 Wang J, Hu Y, Wang Y, et al. Parenting stress in Chinese mothers of children with autism spectrum disorders. Soc Psychiatry Psychiatr Epidemiol 2013;48:575-82.

37 Liu L, Wang M. Parenting stress and children's problem behavior in China: the mediating role of parental psychological aggression. $J$ Fam Psychol 2015;29:20-8.

38 Zung WW. A rating instrument for anxiety disorders. Psychosomatics 1971;12:371-9.

39 Zung WW. A self-rating depression scale. Arch Gen Psychiatry 1965;12:63-70.

40 Zung WW. The measurement of affects: depression and anxiety. Psychological measurements in psychopharmacology. Karger Publishers, 1974: 170-88.

41 Cox JL, Holden JM, Sagovsky R. Detection of postnatal depression. development of the 10-item Edinburgh postnatal depression scale. Br J Psychiatry 1987;150:782-6.

42 Heh SS. Validation of the Chinese version of the Edinburgh postnatal depression scale: detecting postnatal depression in Taiwanese women. Hu Li Yan Jiu 2001;9:105-13.

43 Belfort MB, Rifas-Shiman SL, Sullivan T, et al. Infant growth before and after term: effects on neurodevelopment in preterm infants. Pediatrics 2011;128:e899-906.

44 Serenius F, Källén K, Blennow M, et al. Neurodevelopmental outcome in extremely preterm infants at 2.5 years after active perinatal care in Sweden. JAMA

45 Larroque B, Ancel P-Y, Marret S, et al. Neurodevelopmental disabilities and special care of 5-year-old children born before 33 weeks of gestation (the EPIPAGE study): a longitudinal cohort study. The Lancet 2008;371:813-20.

46 Ishii N, Kono Y, Yonemoto N, et al. Outcomes of infants born at 22 and 23 weeks' gestation. Pediatrics 2013;132:62-71.

47 Moore T, Hennessy EM, Myles J, et al. Neurological and developmental outcome in extremely preterm children born in England in 1995 and 2006: the EPICure studies. BMJ 2012;345:e7961.

48 Pierrat V, Marchand-Martin L, Arnaud C, et al. Neurodevelopmental outcome at 2 years for preterm children born at 22 to 34 weeks' gestation in France in 2011: EPIPAGE-2 cohort study. BMJ 2017;358:j3448.

49 Simões RV, Muñoz-Moreno E, Cruz-Lemini M, et al. Brain metabolite alterations in infants born preterm with intrauterine growth restriction: association with structural changes and neurodevelopmental outcome. Am J Obstet Gynecol 2017;216:62. e1-62.e14.

50 Chang J-H, Hsu C-H, Tsou K-I, et al. Outcomes and related factors in a cohort of infants born in Taiwan over a period of five years (20072011) with borderline viability. J Formos Med Assoc

51 Abbott A. Neuroscience: the brain, interrupted. Nature 2015;518:24-6.

52 Peralta-Carcelen M, Schwartz J, Carcelen AC, ACJCip C. Behavioral and socioemotional development in preterm children. Clin Perinatol 2018;45:529-46. 